ARTICLE

\title{
Development of a Geometry-Coupled Visual Analysis System for MCNP
}

\author{
Pengcheng $\mathrm{LONG}^{1,2, *}$, Qin $Z \mathrm{ZENG}^{1,2}$, Tao HE ${ }^{2}$,Junjun ZHANG ${ }^{1}$, \\ Dongchuan $\mathrm{YING}^{1}$, Shaoheng $\mathrm{ZHOU}^{3}$ and Yican WU ${ }^{1,2}$ \\ ${ }^{1}$ Institute of Plasma Physics, Chinese Academy of Sciences (ASIPP), \\ P.O. Box 1126, Hefei, Anhui, 230031, China \\ ${ }^{2}$ School of Nuclear Science and Technology, University of Science and Technology of China (USTC), \\ P.O. Box 6022, Hefei, Anhui, 230029, China \\ ${ }^{3}$ School of Mathematics, Hefei University of Technology (HFUT), Hefei, Anhui, 230009, China
}

\begin{abstract}
MCNP is a general and powerful Monte Carlo code for photons, neutrons, and electrons transport simulation and is widely used in nuclear analysis. However, external specialized data analysis codes may be needed to analyze complex MCNP calculation results. Especially, there is a lack of efficient data analysis codes which supports geometry-coupled visual analysis. To address the post-processing concerns, a geometry-coupled visual analysis system for MCNP, was developed with 3D scientific visualization and graphics processing unit programming technologies by FDS Team. It was designed and implemented to provide friendly graphical user interface based data manipulation, 3D plotting of tally results, and visual data analysis coupled with geometries. Testing and preliminary application results showed that the system provided a satisfied visual analysis approach for the post-processing of MCNP and obviously enhanced the analysis efficiency.
\end{abstract}

KEYWORDS: MCNP, post-processing, visual analysis, graphics processing unit

\section{Introduction}

The Monte Carlo N-Particle transport code (MCNP), ${ }^{1)}$ developed by the U. S. Los Alamos National Laboratory, is a general and powerful Monte Carlo code for photons, neutrons, and electrons transport simulation. Because of its strong adaptability in complex geometry and high precision in the calculations when it is used in the analysis of nuclear physics, it has been widely applied in the reactor physical design, radiation protection, nuclear detection, aerospace and other fields. However, external specialized data analysis codes may be needed to analyze complex MCNP calculation results. Especially, there is a lack of efficient data analysis codes which supports geometry-coupled visual analysis. To address the post-processing concerns, a large number of research activities have been carried out. In Reference 2, automatic data extraction and curve plotting are achieved by modifying the MCNP code to insert positioning key words into the output data files. In Reference 3, a visual analysis system for MCNP voxel data is presented. In Reference 4, a visual data analysis approach coupled with geometries is presented. The calculation results could be mapped onto the computing geometry surfaces with the help of external finite element analysis code ABAQUS and data visualization code VisIt. But this approach is depend on commercial tool ABAQUS and associated user programming and it's too sophisticated. Overall, there is still lack of specialized and efficient visual analysis system for MCNP post-processing, especially for intuitively visualized

\footnotetext{
*Corresponding author,E-mail:pchlong@ipp.ac.cn
}

(C) 2011 Atomic Energy Society of Japan, All Rights Reserved. analysis of three-dimensional results with computational geometries.

To address the post-processing concerns, a geometry-coupled visual analysis system for MCNP (MCNPViz), was developed by FDS Team. This system is a sub module of the CAD-based multi-functional 4D neutronics simulation system VisualBUS, ${ }^{5,6)}$ which was integrated with automatic modeling, calculation and visual analysis functions. In VisualBUS, the calculation modeling capability for MCNP is supported by MCAM (Monte Carlo Automatic Modeling Program for Radiation Transport Simulation), ${ }^{7-9)}$ which supports the bi-directional automatic conversion between CAD models and MCNP models. The visualization functions of VisualBUS are supported by the subsystem SVIP-N (Scientific Visualization Program for Neutronics). ${ }^{10,11)}$ The work in this paper is under the framework of SVIP-N and is designed to support specialized and efficient visual analysis for MCNP post-processing. It was implemented with $3 \mathrm{D}$ scientific visualization, graphics processing unit based visual programming and the MCNP geometry reversion technologies which is adopted in MCAM. And it was designed and implemented to provide friendly graphics user interface based data manipulation, 3D plotting of tally results, and visual data analysis coupled with geometries.

In this paper, the system architecture and overall design of MCNPViz is presented in Section II, and main capabilities and features of the system are introduced in Section III. In Section IV, the testing based on Experimental Advanced Superconducting Tokamak EAST is described. ${ }^{12)}$ Finally, a brief summary is given in Section V. 


\section{System Architecture and Design}

MCNPViz is designed to make use of advanced scientific visualization technology to provide automatic, visual, intuitive and efficient post-processing environment for MCNP. The system was designed according to object-oriented concept and modular method and was implemented under a strict process management in accordance with modern software engineering methodology. System architecture is shown in Fig. 1, which expresses the system's interaction with the external data and the cooperative relations between internal modules. The system modules are as follows:

(1) Physical data extraction module: automatically parses, classifies and extracts various useful physical data from MCNP calculation result files. It supports not only basic tally results (such as surface tally, cell tally, etc.), but also mesh tally results. For basic tally results, physical data links with tally surfaces or cells. And data correspond to grid mesh for mesh tally results.

(2) Cell geometry extraction module: automatically reconstruct the computational geometry described in the beginning of result files. And the restructured geometries is used to analysis the results.

(3) Data management module: extracted data or imported external data are managed in this module. In the module, interfaces for import or export data at various formats (such as, binary or ASCII raw data format, VTI data format) are provided, and the relation between physical data and computational geometry is recorded.

(4) Geometry management module: various types of geometry (such as, tally surfaces, tally geometries, other computational geometries and external imported geometry) are managed in this module. Various interfaces for import or export geometries are also provided.

(5) Data visualization module: various common data visual analysis approaches are provided here, such as iso-contour, iso-surface, cloud mapping, volume visualization and animation. Especially, the volume visualization is very useful for analysis of spatial mesh tally data.

(6) Geometry visualization module: geometry display at various styles, such as point cloud, wireframe and surface shaded, is supported by this module. And the interface for geometrical attributes (such as color, opacity and visibility) of each geometry is provided in this module.

(7) Data visualization coupled with geometry module: this module provides the features to analyze all kinds of data coupled with geometries. Mapping data onto arbitrary geometry surface is supported. The data visualization and the geometry display could be rendered in the same scene at the same time by using transparent technology. Furthermore, the data visualization scene could be cut by geometry. So flexible and fine visual data analysis could be performed by using

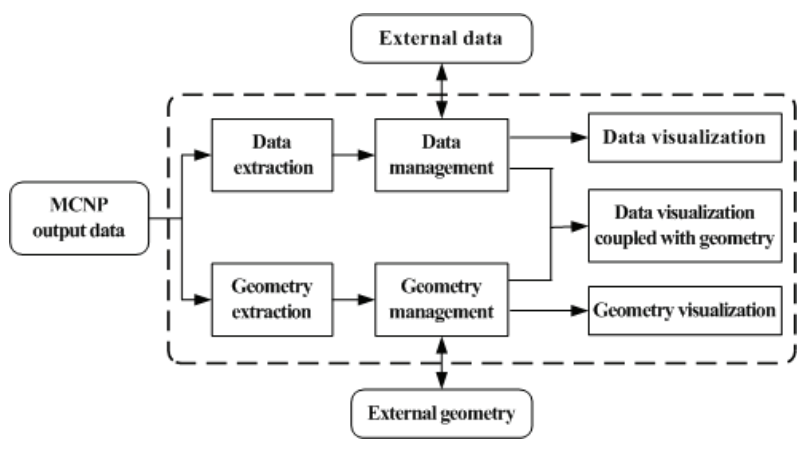

Fig. 1 System Architecture

these advanced visual analysis capabilities.

\section{Main Capabilities and Features}

(1) Numerical processing and analysis based on friendly graphical user interface: the system supports a variety of numerical processing and analysis capabilities based on friendly graphical user interface. So users could complete the main numerical processing and analysis simply by the way of mouse clicks, and don't need to directly face to the complicated original calculation files with huge amounts of information.

(2) Visual analysis of multidimensional data: the system supports image-based visual data analysis as the main way. It provides various styles of visualization, such as curve (such as energy group based flux data distribution curve), iso-contour, iso-surface, cloud mapping, volume visualization of data spatial distribution. The system does not only enhance the efficiency of data analysis, but also enrich data analysis tools. For basic tally results, only energy group based physical data distribution curve is supported due to the characteristic of the data. But all styles of visualization can be used for mesh tally results.

(3) Geometry-coupled visual analysis of $3 D$ data:

- Geometry-coupled visual analysis of cell tally results and surface tally results: to show the result data of tally cells or tally faces, the system maps the data into pseudo-color, uses the color as the attribute of tally cell or surface and display the corresponding geometries in order to achieve an intuitive data analysis.

- Geometry-coupled visual analysis of mesh tally results: the mesh tally result data shows a $3 \mathrm{D}$ distribution. To characterize the relationship between the spatial data distribution and computational geometry, the system introduces visual process of data and provides three methods of geometry-coupled visual analysis: mapping data onto arbitrary geometry surface, mixed rendering of data visualization and geometry in the same scene at the same time, and cutting visualization based on geometry. Mapping data onto arbitrary geometry surface refers to map the data into pseudo-color as the color texture of computational 
geometry, so that the distribution of the geometry surface's data can be reflected when geometry is displayed. Mixed rendering of data visualization and geometry refers to show of geometric model during the data visualization and assists to analyze data distribution through the relationship between geometry and data distribution. Cutting visualization based on geometry is that users can cut out (or cut off) data within interested geometries, so that system achieves more flexible, sophisticated visual analysis on local data. These geometry-coupled data visualization algorithms are based on scientific visualization's and GPU visualization's programming technology. These algorithms will be described in later articles.

(4) Automatic, visual and integrated analysis environment for MCNP calculation results: the system provides a set of integrated analysis environment for post-processing of MCNP calculation results. Also the system can effectively meet the current demand for the analysis of MCNP calculation results and complete multiple styles of visual data analysis automatically and quickly in a small amount of interaction.

\section{Testing}

Lots of testing cases, to verify the validity and practicability of the system, have been conducted. Testing platform was ordinary personal computer with CPU of CORE 2 DUO 2.66 GHz, GPU of Geforce 9300GE. The numerical results has been compared to those obtained from traditional manual process method, and part of the visualization results has been compared to those generated in Tecplot software, ${ }^{14)}$ which is a popular and powerful data analysis and visualization software. Real-world examples, such as FDS-I and EAST have been adopted. FDS-I is one kind of fusion driven subcritical system. ${ }^{13)}$ In this paper, the detailed testing case based on EAST will be introduced as following.

EAST (Experimental Advanced Superconducting Tokamak) is the first non-round cross section complete superconducting fusion experimental Tokamak device built at ASIPP. A $45^{\circ}$ section of EAST CAD model, as shown in Fig. 2, has been chosen for testing analysis.

In order to verify the correctness of the system, the visualization results of the system were compared with Tecplot. Due to the disadvantage of Tecplot, cross-section visualization function is selected to compare. Figures 3 and $\mathbf{4}$ show that cross-section visualization of neutron flux at the vertical plane of $-22.5^{\circ}$ in SVIP-N has a good agreement with the result in Tecplot.

Volume visualization and data visualization coupled with geometry are not supported by Tecplot. So verification of these capabilities is conducted by experts. One scenario about 3D plotting of mesh tally results in spatial distribution is shown in Fig. 5. The trend of data distribution is expressed by the pseudo-color map and agrees well with the theoretical analysis. Furthermore, the capability to map data onto computational geometry is demonstrated in the figure. The EAST machine is placed in the space indicated in

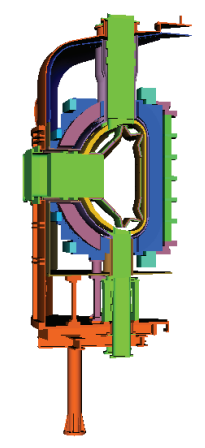

Fig. $245^{\circ}$ section of EAST CAD model

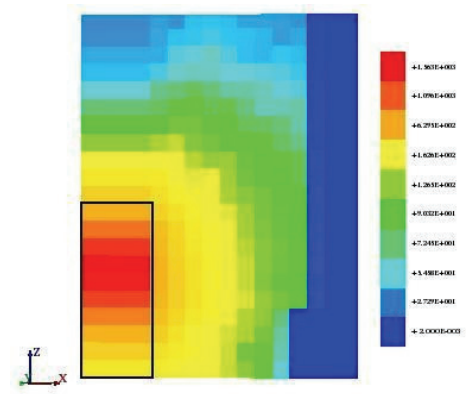

Fig. 3 Cross-section visualization of neutron flux in SVIP-N

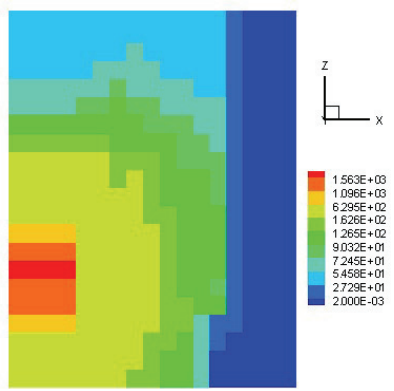

Fig. 4 Cross-section visualization of neutron flux in Tecplot

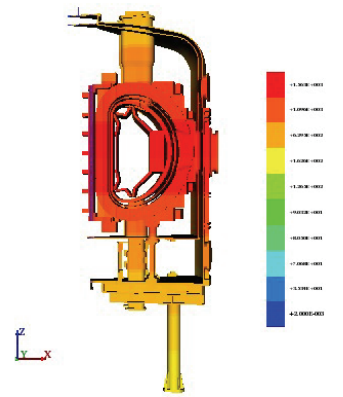

Fig. 5 Mapping 3D neutron flux onto geometry surfaces

Fig. 3 with black wire frame. The visualized analysis procedure is as follow: first, import the 3D MCNP mesh tally results into SVIP-N; secondly, resample the original mesh data into regulation data; thirdly, import the computational geometry into SVIP-N and finally select the regulation data as the 3D texture of the computational geometries to generate Fig. 5 . 


\section{Summary}

With the maturity and widespread use of MCAM, nuclear analyses of complex problems are increasing, and the 3D mesh tally is gradually used, conventional treatment of MCNP has gradually become the bottleneck which is restricting MCNP. For such problems, this paper addresses the visual analysis of 3D data and the visualization methods coupled with geometry. A geometry-coupled visual analysis system for MCNP (MCNPViz), was developed with 3D scientific visualization and graphics processing unit programming technologies by FDS Team. With MCNPViz, advanced visual data analysis capabilities for Monte Carlo and Discrete Ordinates (SN) based transport simulation results are support by SVIP-N. An integrated platform for visual and automatic transport simulation is established with the help of SVIP-N, MCAM and SNAM (SN Automatic Modeling Program for Radiation Transport Simulation). ${ }^{15,16)}$

\section{References}

1) T. Goorley, J. Bull, F. Brown et al., Release of MCNP5_RSICC_130, LA-UR-04-4519, Los Alamos National Laboratory (LANL) (2004).

2) F. Jiajin, W. Yi, C. Jianping et al., "Development of visual platform of MCNP4B," Nucl. Electron. Detect. Technol., 22[1], 52-55 (2002).

3) Y. Huang, J. Kim, R. G. Moreira et al., "A web-based information system for MCNP simulation of irradiation of complex shaped foods," Appl. Eng. Agr., 24[2], 233-242 (2008).

4) J. R. Parry, J. A. Galbraith, "Visualizing MCNP Tally Segment Geometry and Coupling Results with ABAQUS," Trans. Am. Nucl. Soc., 97, 514-516 (2007).
5) Y. Wu, J. Li, Y. Li et al., "An integrated multi-functional neutronics calculation and analysis code system: VisualBUS," Chinese J. Nucl. Sci. Eng., 27[4], 365-373 (2007).

6) Y. Wu, L. Hu, P. Long et al., "Development of design and analysis software for advanced nuclear systems", Chinese $J$. Nucl. Sci. Eng., 30[1], 42-50 (2010).

7) $\mathrm{Y} . \mathrm{Wu}$, "CAD-based Interface Programs for Fusion Neutron Transport Simulation," Fusion Eng. Des., 84[7-11], 1987-1992 (2009).

8) Y. Wu, Y. Li, L. Lu et al., "Research and development of the automatic modeling system for Monte Carlo particle transport simulation," Chinese J. Nucl. Sci. Eng., 26[1], 20-27, 78 (2006).

9) Y. Li, L. Lu, A. Ding et al., "Benchmarking of MCAM 4.0 with the ITER 3D model," Fusion Eng. Des., 82[15-24], 2861-2866 (2007).

10) Y. Luo, P. Long, Y. Xue et al., "Development of integrated visualization platform SVIP for neutronics analysis," Chinese J. Nucl. Sci. Eng., 27[4], 374-378 (2007).

11) Y. Luo, P. Long, G. Wu et al., "SVIP-N 1.0: An integrated visualization platform for neutronics analysis," Fusion Eng. Des., 85[7-9], 1527-1530 (2010).

12) W. Wu, S. Wu, J. Yu et al., "Assembly of the Super-conducting Tokamak EAST," J. Kor. Phys. Soc., 49, 14-17 (2006).

13) Y. Wu, FDS Team, "Conceptual design activities of FDS series fusion power plants in China," Fusion Eng. Des., 81[23-24], 2713-2718 (2006).

14) TecPlot, http://www.tecplot.com/

15) H. Hu, Y. Wu, M. Chen et al., "Benchmarking of SNAM with the ITER 3D model," Fusion Eng. Des., 82, 2867-2871 (2007).

16) P. Long, J. Zou, S. Huang et al., "Development and application of SN Auto-Modeling Tool SNAM 2.1," Fusion Eng. Des., 85[7-9], 1113-1116 (2010). 